\title{
A new fourth-order explicit Runge-Kutta method for solving first order ordinary differential equations
}

\begin{abstract}
We present a new form of fourth-order explicit Runge-Kutta method in which the terms $\mathrm{f}$ and fNgre used in the formulae to increase the order as well as to enhance the performance of the method. The proposed method is then used to solve first order ordinary differential equation of the form $y^{\prime}$ (IfIf(t, $\left.y(t)\right)$. From the numerical results, it is observed that the new method is more efficient in terms of time needed for solving the problems while maintaining the same accuracy as the existing Runge-Kutta method of the same order. Stability of the method is discussed the region of absolute stability is shown.
\end{abstract}

Keyword: Absolute stability; Explicit; Runge-Kutta 